\title{
An affinity score for grains merging and touching grains separation
}

\author{
T. Chabardès, P. Dokládal, M. Faessel, M. Bilodeau \\ PSL Research University - MINES ParisTech \\ CMM, Center for Mathematical Morphology \\ 35 rue Saint Honoré - Fontainebleau, France
}

\begin{abstract}
The physical properties of granular materials on a macroscopic scale derive from their microstructures. The segmentation of CTimages of this type of material is the first step towards simulation and modeling but it is not a trivial task. Non-spherical, elongated or nonconvex objects fail to be separated with classical methods. Moreover, grains are commonly fragmented due to external conditions: aging, storage conditions, or even user-induced mechanical deformations. Grains are crushed into multiple fragments of different shape and volume; those fragments drift from one another in the binder phase. This paper focuses on reconstruction of grains from these fragments using scores that match the local thickness and the regularity of the interface between two objects from a given primary segmentation of the material. An affinity graph is built from those scores and optimized for a given application using a user-generated ground truth on a $2 \mathrm{D}$ slice of the tridimensional structures. A minimum spanning tree is generated, and a hierarchical cut is performed. This process allows to reassemble drifted fragments into whole grains and to solve the touching grains problem in tridimensional acquisitions.
\end{abstract}

\section{Introduction}

X-Ray microtomography is now widely used by professionals and non-experts to characterize the structures of materials of interest. However, assessing precisely the structural information by conventional means is difficult due to the size of the acquisitions. Human interactions are unpractical for this amount of data. There is a need for automatic methods of analysis that can harvest the ever-increasing computing power.

This study focuses on composite materials made from a rubbery binder and brittle grains of various shapes and sizes mixed into a homogeneous mixture. Damage occurs when a mechanical shock is applied, causing fragmentation of these grains. The damaged structure affects the behavior of the material. Evaluating the extent of fragmentation may be useful for estimating effects on the reaction of the mixture. Highly clustered fragments and complex shapes require specific methods to identify all connections. The drift of fragments in the binder phase can not be solved using a method only based on the barycenter distance, as e.g. in [11], see figures $(1,6)$. 


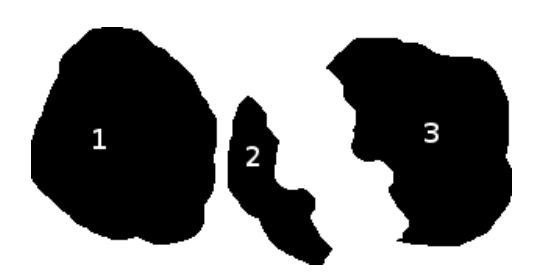

Fig. 1. The fragment 2 is closer to 1 even though it should be associated to 3 .

In the first part, we present how to evaluate the shape of the interface between two objects of a given segmentation. Pixels equidistant to two objects border to border forms separation segments. Each pixel of those segments is valued by the distance to border. Measurements are performed on the pixels along the segments such as mean, standard deviation of the pixels value. Some already relate to affinities; others can be transformed to affinities using a Gaussian kernel. In a second part, the grains are reassembled from the derived fragments using the proposed affinity score in combination with a hierarchical clustering, and a set of predetermined measurements done on the unprocessed acquisition.

\subsection{Materials and 3D images.}

The studied materials are composite materials with an elastic binder degraded by a mechanical impact of a falling mass. Those samples were acquired by Xray microtomography with a Skyscan 1172 high-resolution micro-CT system. Images of the work of Gillibert were recovered for comparison purposes [6]. The introduced method was also tested on our acquisitions. The following acquisitions illustrated in the figure (2) are studied:

- MAT1 is a $1014^{*} 1155^{*} 250$ voxels image $\left(3650.4^{*} 4158^{*} 900 \mu \mathrm{m}^{3}\right)$. A mechanical impact is exerced from a $2 \mathrm{~kg}$ mass falling $15 \mathrm{~cm}$. This material has been studied in [8].

- MAT2 is a $1000^{*} 1000^{*} 1000$ voxels image $\left(2097^{*} 2097^{*} 2097 \mu \mathrm{m}^{3}\right)$. A mechanic impact is exerced from a $2 \mathrm{~kg}$ mass falling $30 \mathrm{~cm}$. This is a new material.

\subsection{Initial segmentation.}

The work presented in this paper requires a primary segmentation on the acquisition. We suppose each fragment for every grain isolated, which effectively induces an over-segmentation, where grains are fragmented. Then, we successively merge pairs of fragments, whose affinity is the highest. Several approaches can be considered to create this over-segmentation. Gillibert and al. used a multiscale stochastic watershed in [7]. The estimation of the granulometry makes it possible to select appropriate scales to perform several stochastic watersheds and combine them to obtain a correct segmentation for each size of grains. 


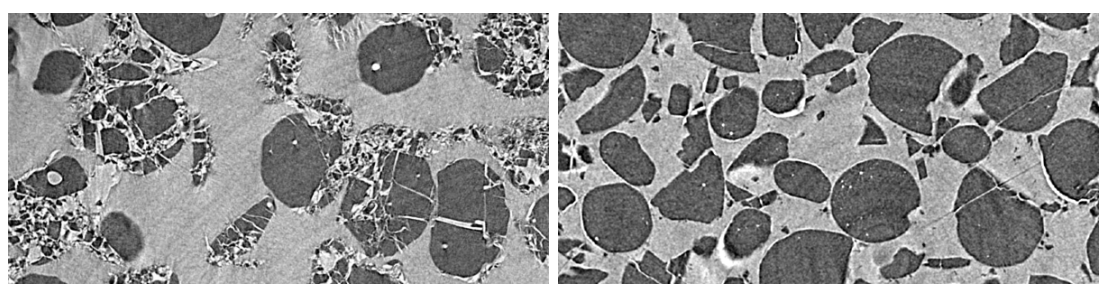

(a) MAT1

(b) MAT2

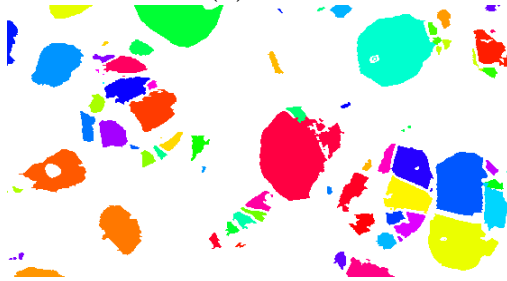

(c)

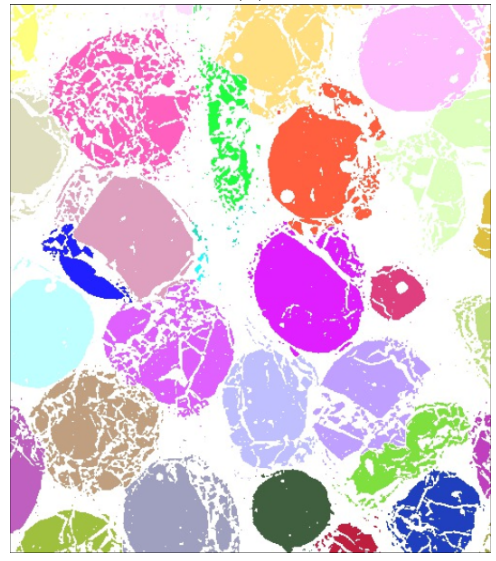

(e)

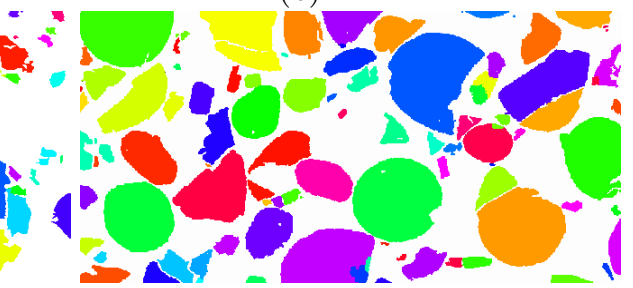

(d)

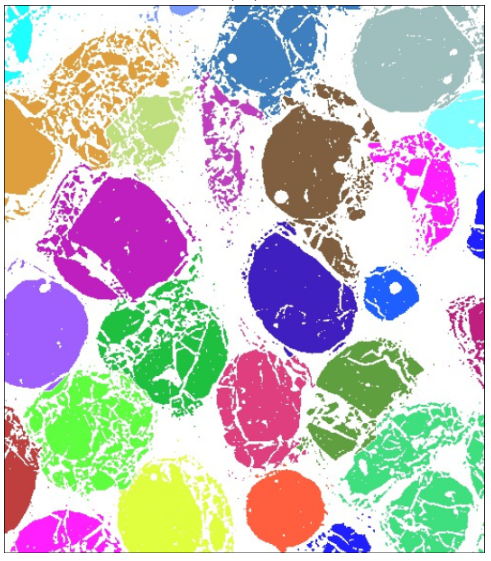

(f)

Fig. 2. (a,c) are 2D slices of 3D X-ray microtomographic images of fragmented granular material. $(\mathrm{b}, \mathrm{d})$ are the corresponding over-segmentation produced by h-minima markers. (e,f) are results obtained in [8]. (e) Watershed segmentation of the closed image after a h-minima filter. (f) Watershed segmentation using markers computed from the K-means. In (e) and (f), we can observe several grains over-segmentated or under-segmentated.

However, this approach is tedious and costly. A less refined approach can be used, as over-segmented fragments will be merged during the clustering process: the resulting affinity will be high in this case. In this study, the image is first binarized using a method close to the Otsu threshold. The watershed is then computed from the inverse of the distance map, and the set of markers is generated by using the $h$-minima filter.

Other approaches could also be considered, such as using the distance map on the binarized image, as a probability density map to generate random markers 
for the watershed transformation. The only requirement is to find a right amount of over-segmentation. Every fragment can be split into a small number of objects in the over-segmentation, but no object must gather more than one fragment. In the figure (2), two segmentations have been provided for the studied materials.

\subsection{State of art of fragments merging.}

A morphological approach for removing cracks is to use the morphological closing combined with a volumic opening, see $[12,18]$. Small connected components are therefore removed, and fragments close in space are merged. The constrained watershed transform introduced by [2] used on the closed image can differentiate a fragmented grain from two grains merging. Markers have to be chosen to represent each grain appropriately and are an important parameter in this method. Two approach exists. The first approach is topological and uses the h-minima filter introduced in [19]. The use of the h-minima with the watershed on the distance map is standard, but if the grains are highly fragmented and the fragments are scattered, the algorithm fails to reconstruct grains accurately.

The second approach is based on a method of cluster analysis, the K-means clustering, which aims to partition a set of observations into K clusters, as described in [11]. The number of clusters can be automatically calculated from a covariance measure. In [8], the K-means transform is used to generate the appropriate markers for the watershed transformation.

We propose in section 3 a fully automatic method for homogeneous granular material, which can be adapted to heterogeneous material. Based on the previously described affinity score, a maximum spanning tree is built. This maximum spanning tree can be used to form a hierarchy between fragments represented as a dendrogram. The estimation of grains size is used to cut this hierarchy and produce the final result.

\subsection{State of art of touching objects separation.}

Separation of touching objects is a recurring problem in image processing. Classical techniques like Hough-transform [10] or the watershed applied to the inverse of the distance function performs well when the objects of interest possess regular shapes such as spheres. Unfortunately, when shape and size of objects vary considerably or when clusters contain many objects, classical methods may fail to produce the desired separation. Differents approach exists [16]: morphologybased procedures [1], contour-based techniques [15], graph theoretic approaches, parametric fitting algorithms and level set approach [3,17]. Morphological multiscale decomposition can decompose clusters into size-specific scales, carrying markers for each disjoint region [9]. Methods exist for shape specific objects. In [20], a method based on a modified version of the pseudo-Euclidean distance transformation is able to split fused ellipses. In [5], a gap-filling method is proposed for elliptic shapes. 


\section{The morphological affinity score for adjacent objects.}

\subsection{Definitions.}

We start from an initial segmentation as described in section 1.2. Let $f: \mathscr{D} \rightarrow$ $\{0,1\}$ a binary image, where $\mathscr{D}$ is the definition domain.

Binary objects We define the set $\mathscr{X}$ of $N$ objects as $\mathscr{X}=\left\{X_{i}\right\}_{i<N}$, so that $X_{i} \subset \mathscr{D}$ and $X_{i} \cap X_{j}=\emptyset$ for $i \neq j$. Each $X_{i}$ represents a grain or a fragment. The complement $D \backslash \mathscr{X}$ is the binder.

Distance mapping We define the distance mapping $d: \mathscr{D} \rightarrow \mathbb{N}$ labeling each pixel with the distance to the nearest object $X_{i} \in \mathscr{X}$ according to a chosen metric.

SKIZ by distance function A zone of influence of a object $X_{i}$ of $\mathscr{X}$ is the subset of $\mathscr{D}$ that are closer to $X_{i}$ than to any other object, and we note it $z i(i)$. A SKIZ is defined as the boundary of all zones of influence. We compute the SKIZ by using the watershed transform. We note this set $\operatorname{skiz}(\mathscr{X})$ and we assume it thin and connected using some connexity $\complement$.

Triple points The set $\mathscr{T}$ of triple points is the set of pixels that are equidistant to three or more distinct objects. We have extended the set $\mathscr{T}$ by including pixels on the border of $f$ that are equidistant to two objects. The triple points are the extremities of a given segment of the SKIZ.

Interfaces We define interfaces between $X_{i}, X_{j}$ as part of the segments along the SKIZ. We note $I$ the set of interfaces, and $I_{i j}$ the interface between two distinct objects $X_{i}$ and $X_{j}$. The distance decreases when going from triple points inwards, as shown in figure (4). However, this distance behaves differently when observed closer to the objects of interest. We define an incremental process to thin the skiz into the non-decremental segment.

$$
\begin{aligned}
& I^{0}=\operatorname{skiz}(\mathscr{X}), T^{0}=\mathscr{T} \\
& I^{i+1}=I^{i} \backslash T^{i+1} \\
& T^{i+1}=\left\{a \mid \exists b \complement a, b \in T^{i}, d(b)>d(a)\right\}
\end{aligned}
$$

Finally, we define $I_{i j}=I^{\infty} \cap \mathrm{zi}(i) \cap \mathrm{zi}(j)$. Those interfaces are the support for building our new affinity scores. The distance mapping considered along the pixels of an interface gives various information regarding the shape of the interface between two objects. The figure (3.d) illustrates the interfaces $I_{i j}$ between various objects given some initial segmentation (3.b).

\subsection{Weigths.}

In the following part, we propose several measures to weight the interfaces. All proposed weights are $w: I \rightarrow \mathbb{R}$. However, it is important to notice that the range of $w$ differs regarding to the considered weight. 


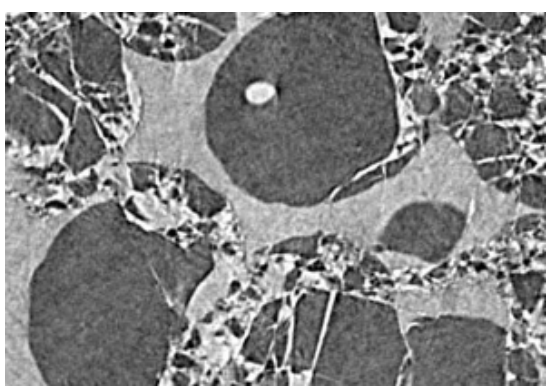

(a) original

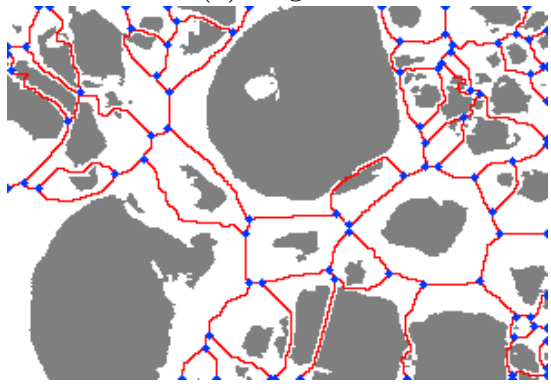

(c) skiz and triple points

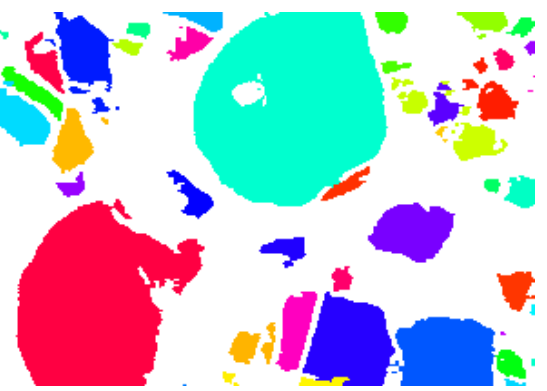

(b) over-segmentation

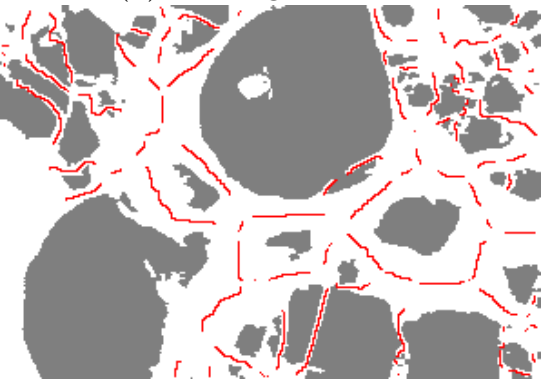

(d) thin interfaces

Fig. 3. The process to generate interfaces is illustrated. In (a) a granular material. (b) an example of segmentation that was used to produce the skiz. In (c), The skiz is drawn in red, the objects in gray, and triple points in blue. In (d) are shown interfaces generated using the Manhattan distance.

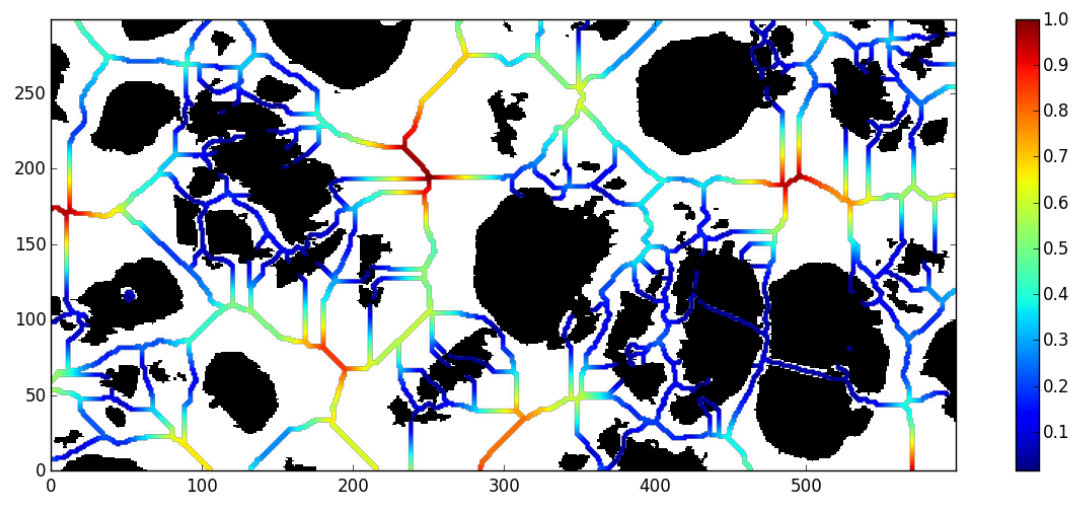

Fig. 4. Divergence of the distance along the skiz is observed towards the triple points.

The barycenter distance. The simplest measure already used in [8] is the Euclidean distance between barycenters of two distinct objects: $w_{b a r}\left(I_{i j}\right)=$ $\left\|\overline{X_{i}}-\overline{X_{j}}\right\|_{L^{2}}$, where $\bar{X}$ denotes the barycenter of the object labeled $X$ in $\mathscr{X}$. 
Sum on the pruned interface: $w_{d}\left(I_{i j}\right)=\sum_{x \in I_{i j}} d(x)$ with $d$ the distance to fragments.

Length of the pruned interface: $w_{l}\left(I_{i j}\right)=\left|I_{i j}\right|$ the length of each interface.

Mean: $w_{\mu}\left(I_{i j}\right)=w_{d}\left(I_{i j}\right) / w_{l}\left(I_{i j}\right)$ the mean of the distance.

Variance: $w_{\sigma^{2}}\left(I_{i j}\right)=\sum_{x \in I_{i j}} d(x)^{2}-w_{\mu}^{2}\left(I_{i j}\right)$ the variance of the distance.

\subsection{Affinities.}

We note $A$, a positive and symetric matrix, with $a_{i j} \in(0,1)$ the affinity score of $X_{i}$ and $X_{j}$, where a value close to 1 corresponds to two closely related objects and a value close to 0 to distinct objects. All previous weights can be transformed to an affinity. We use a gaussian kernel for all weights that relate to a distance, where $\sigma_{w}$ is the scale of the kernel, as follows:

$$
A=G\left(w, \sigma_{w}\right)=\frac{1}{\sqrt{2 \pi} \sigma_{w}} e^{-\frac{w^{2}}{2 \sigma_{w}^{2}}}
$$

Using the previous equation, we have $A_{\mathrm{bar}}, A_{d}, A_{l}, A_{\mu}, A_{\sigma^{2}}$.

Length-to-surface ratio. Other affinities can be obtained from the previous weigths. Below, we use an affinity based on the surface of the objects. We define $s(X)$ the surface of the object $X$ as $s(X)=|\{x, x \in G(f), x \in X\}|$, where $G(f)$ is a morphological gradient of the image $f$. We now define the new affinity $A_{s}$ as follows:

$$
\left(a_{s}\right)_{i j}=\frac{w_{l}\left(I_{i j}\right)}{\min \left(s\left(X_{i}\right), s\left(X_{j}\right)\right)}
$$

Note that $w_{l}\left(I_{i j}\right)$ can be bigger than the surface of an objects, and the resulting affinity has to be bounded to $(0,1)$.

All those affinities can be used as such with clustering techniques. However, those affinities complement each other and we can combine several affinities as follows:

$$
A=\sum_{k} A_{k} f_{k}
$$

In the next section, we used a linear combination of the affinities $A_{\mathrm{bar}}, A_{\mu}$, $A_{\sigma^{2}}, A_{s}$ to merge fragments in a composite material fragmented by mechanical pressure. We have optimized the linear combination by minimizing a score between the resulting merging and a ground truth, as described in section 3.2. 


\section{Fragments merging.}

\subsection{Minimum spanning tree and hierarchical cut.}

We can use the linear combination of the introduced affinities with several existing clustering methods, such as K-means, spectral clustering, DBSCAN and other. The inconvenience is when the number of fragments increases computational issues appear (running time, instability of convergence, memory consumption). For given materials, we have chosen a hierarchical clustering and we perform a hierarchical cut using an estimated size of grain extracted from a covariance analysis.

The previous affinity matrix is an adjacency graph. The first step of the proposed clustering method is to produce a hierarchy using the minimum spanning tree of the graph $1-A$ to remove edges of low affinity. We produce a dendrogram from the minimum spanning tree as illustrated in the figure (5). The strategies employed to build the hierarchy can be of any of the commonly used types: agglomerative or divisive.

Cutting the hierarchy depends on the material that is considered. MAT1 is a homogeneous composite with one size of grains. Therefore, the criterium that we used to cut the hierarchy is volumic. The covariance analysis of the raw data can give us an estimate of the mean size of the grains, see [4]. We have associated with each cluster the sum of the volumes of the individual objects composing the former. Cutting the hierarchy can be achieved by the pseudo-code (1).

Due to the previous thinning of the SKIZ, the initial graph might not be connected. The missing edges are diverging all along the SKIZ and therefore of low affinities. Moreover, filtering the graph by removing edges that feature low affinity values can be done to process graphs of lower density. Each connected components can be processed afterward independently.

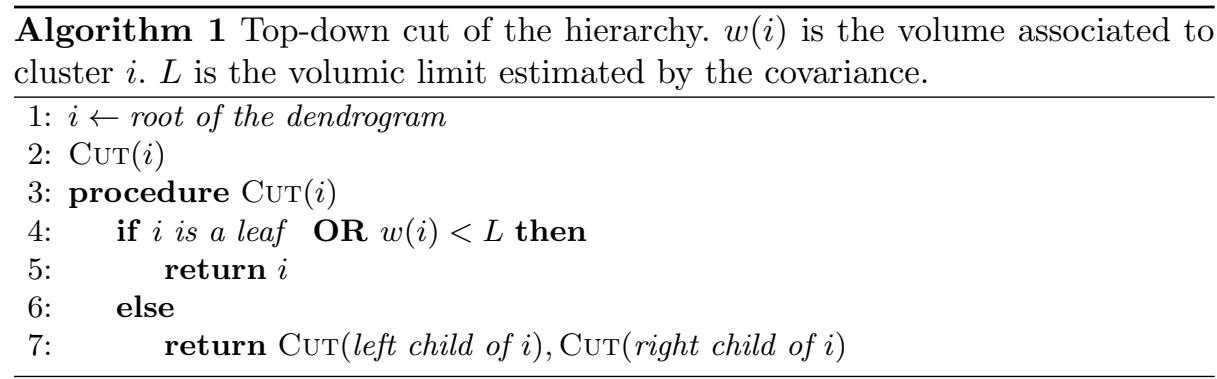

\subsection{Optimizing the affinity}

The choice of the affinity is crucial to obtain the desired result. One can see that a single affinity that was presented earlier is not sufficient to reassemble the 
(a)

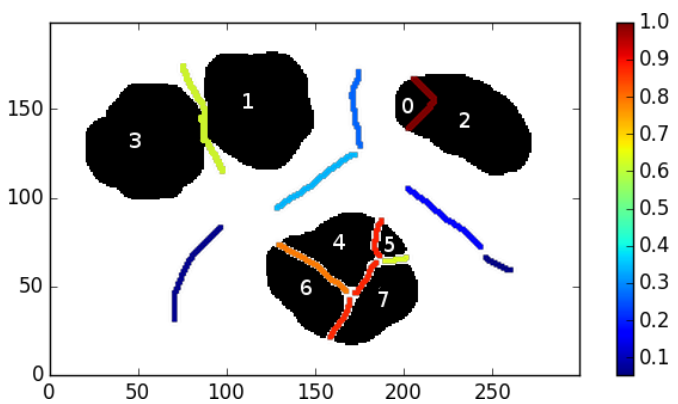

(b)

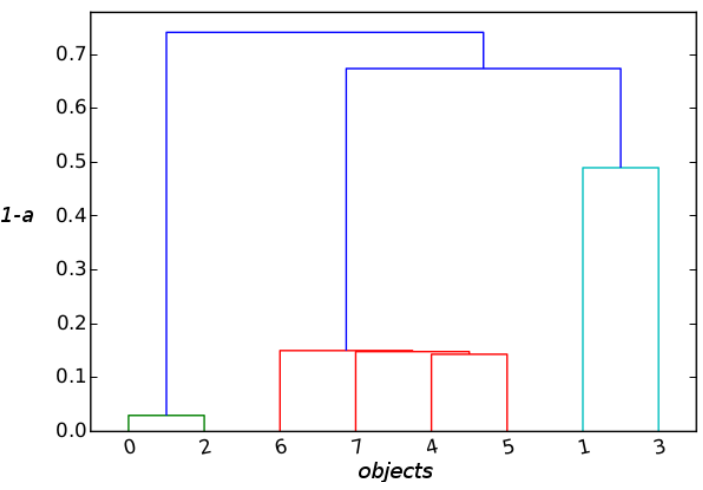

Fig. 5. A simple case of dendrogram built from a maximum spanning tree is illustrated. In (a), Objects are shown in black, and interfaces are valued using a colormap scheme. The affinity used to generate the valued edges is a linear combination of $A_{\mu}, A_{\sigma^{2}}$ and $A_{s}$. The resulting dendrogram is shown in (b), where 3 clusters are visually identified, and the y-axis is $1-A$. We can see that objects 1 and 3 have a lower affinity than 0 and 2 , and are visually two distinct grains that are connected.

fragments, see figure (1). The K-means algorithms is based on the barycenter distance and is not able to reassemble fragments that have drifted far away from their original positions. The size distribution of fragments is wide, the drift between similar fragments can be big enough not to be caught by the naive border-to-border distance as illustrated in figure (6). We assume that a combination of affinities is the key to obtain better results.

We opted for a linear combination of the four affinities $A_{\mathrm{bar}}, A_{\mu}, A_{\sigma^{2}}$ and $A_{s}$. To optimize the affinity, we optimize the segmentation using a user-provided ground truth $(\mathrm{GT})$ on one or more slices of the 3D image.

We construct a bivariate histogram $\mathscr{H}$ from the labels of GT and from the labels obtained by hierarchical clustering extracted from the same slices. $\mathscr{H}=\left[h_{i j}\right]$ is a $M \times N$ sparse matrix where $M$ is the number of detected objects and $N$ the number of objects in the GT. From $\mathscr{H}$, we compute the $F_{1}$ score of the segmentation in the following way: 


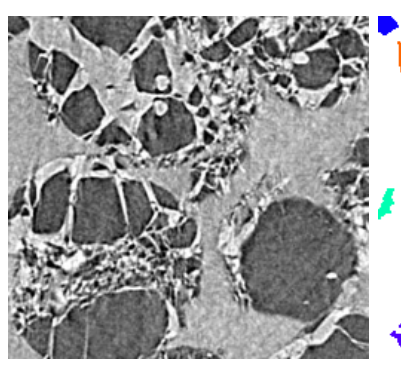

(a) MAT1

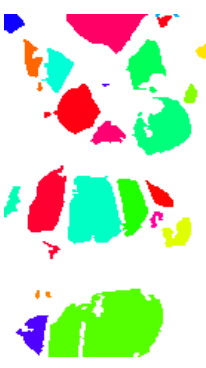

(b)

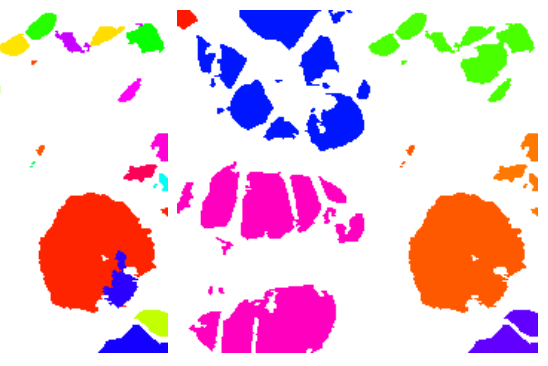

(c)

Fig. 6. (a) shows two fragments that heavely drift from one another on the bottom left. (b) shows the over-segmentation. (c) the result of the fragment merging. The round fragment in (b) in the bottom right corner colored in red in blue, is oversegmented. In (c), the two objects were merged into one orange grain.)

A sum of a line $i=$ const, $P(i)=\sum_{j} h_{i j}$, is the detection rate of label $i$. The index $l=\arg \max _{j} h_{i j}$, is the label corresponding to $i$ in GT. We now compute the true positive $T P(i)=h_{i l}$, the false positive $F P(i)=P(i)-T P(i)$.

The sensitivity and the positive predictive value for every $i$ is: $\operatorname{Sens}(i)=$ $\frac{T P(i)}{P(i)}$ and $P P V(i)=\frac{T P(i)}{T P(i)+F P(i)}$.

A $F_{1}$ score is computed for every detected object, with $H$ the harmonic mean: $F_{1}(i)=H(\operatorname{Sens}(i), P P V(i))$. The overall $F_{1}$ score of the result is the harmonic mean of all scores: $F_{1}=H\left(F_{1}(i)\right), 1 \leq i \leq M$. The vector $\left(f_{k}\right)$ in equation 1 is then determined by minimizing $1-F_{1}$. The minimization method is a modification of Powell's method [13], [14]. It performs sequential one-dimensional minimizations along each vector of the directions set.

\subsection{Results.}

Table (1) shows the size of the generated graph for MAT1 and MAT2. One benefit of this method is to be able to work on a compressed representation of the studied materials from the raw tridimensional data. Further compression is achieved by using a $\epsilon$-filtering of the weighted graph. Here, edges lower than mean $(a)-2 * \operatorname{std}(a)$ have been removed. An appropriate filtering will generate several connected components, which can each be processed independently and in parallel.

Table 1. The graphs generated for MAT1 and MAT2. Here is shown the gain in memory consumption when working on graphs.

\begin{tabular}{|l||l|l|l|l|}
\hline Materials & Number of voxels & Number of objects & Number of edges & After $\epsilon$-filtering \\
\hline MAT1 & $2.9 * 10^{8}$ & 720 & 5106 & 2456 \\
MAT2 & $1.0 * 10^{9}$ & 4660 & 28341 & 12846 \\
\hline
\end{tabular}


Figure (6) shows a crop of a result obtained on MAT1. Figure (7) shows the tridimensional results on MAT1 and MAT2. Some grains are broken down into multiple small fragments, they have been successfully merged. Touching grains have been solved for MAT2 and MAT1 and visually satisfying results have been achieving for the merging of fragments.

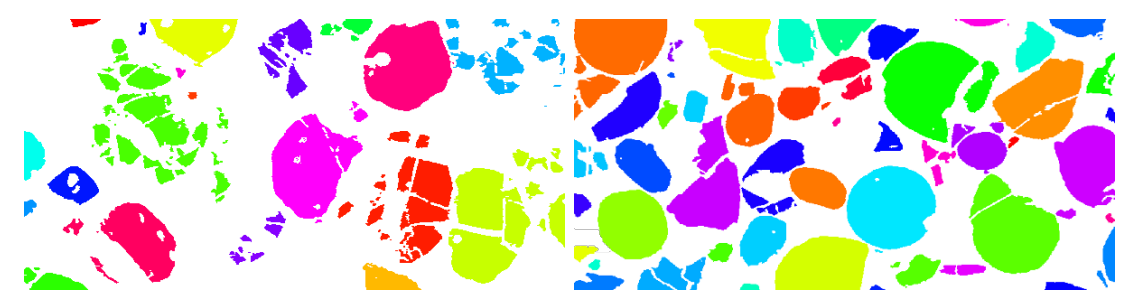

(a) MAT1

(b) MAT2

Fig. 7. Results of the fragments merging method.(a) Slice of reassembled MAT1. (b) Slice of reassembled MAT2. In (a), grains are heavely fragmented such as the green grain in the top-right corner, and heavely clustered such as red and yellow grains in the bottom-right corner. In (b), less fragmentation is observed but there is more connections between grains.

\section{Conclusion}

We have introduced a method to calculate affinities between objects in tridimensional data such as CT images. Those affinities have been applied to the problem of fragments merging using machine learning algorithm. Oversegmented fragments were also merged, as shown in figure (6), while every touching grains remains separated. The touching objects problem were therefor also solved with the same approach. Visually satisfying results were obtained for both, see figure (7). Those affinities are invariant to scales and are able to characterize objects of various shapes. Many perspectives arise from this preliminary work. Further works should first concern the influence of the primary segmentation and the metric employed to build the interfaces. The proposed method for hierarchical clustering is a proof of concept than could be applied to more advanced clustering technics.

\section{Acknowledgment}

This work has received funding from DGA and is a collaboration between Transvalor, DGA, CEA Gramat. The acquisitions are carried out at the CEA Gramat, a french public laboratory affiliated to the Commission of Atomic Energy and Alternative Energies. 


\section{References}

1. S. Beucher. Segmentation d'Images et Morphologie Mathématique. PhD thesis, Ecole Nationale Supérieure des Mines de Paris, jun 1990.

2. S. Beucher and C. Lantuejoul. Use of watersheds in contour detection. International Workshop on Image Processing : Real-time Edge and Motion Detection/Estimation, sep 1979.

3. Eva Dejnozkova and Petr Dokladal. Modelling of Overlapping Circular Objects Based on Level Set Approach, pages 416-423. Springer Berlin Heidelberg, Berlin, Heidelberg, 2004.

4. M. Faessel and D. Jeulin. Segmentation of 3d microtomographic images of granular materials with the stochastic watershed. Journal of Microscopy, 239(1):17-31, 2010.

5. Matthieu Faessel and Francis Courtois. Touching grain kernels separation by gapfilling. Image Analysis and Stereology, 28:195-203, 2009.

6. L. Gillibert and D. Jeulin. Stochastic multiscale segmentation constrained by image content. ISMM'11 Proceedings of the 10th international conference on Mathematical morphology and its application to image and signal processing, pages 132-142, 2011.

7. L. Gillibert and D. Jeulin. Stochastic multiscale segmentation constrained by image content. Soille P, Pesaresi M, Ouzounis GK, eds. Proc 10th int Conf Math Morpho Appl Image Signal Process (ISMM'11). Lect Not Comput Sci, 6671:132-42, 2011.

8. L. Gillibert and D. Jeulin. 3d reconstruction and analysis of the fragmented grains in a composite material. Image Anal Stereal 2013, 32:107-115, 2013.

9. Henk J. A. M. Heijmans. Algebraic framework for linear and morphological scalespaces, 2002.

10. V.F. F. Leavers. Shape Detection in Computer Vision Using the Hough Transform. Springer-Verlag New York, Inc., Secaucus, NJ, USA, 1992.

11. S. P. Lloyd. Least squares quantization. PCM. IEEE Trans Inf Theory, 28:129-137, 82.

12. G. Matheron. Some methods for classification and analysis of multivariate observations. Cam LML, Neyman J, eds. Proc 5th Berkeley Symp Math Stat Probab, 1, 1967.

13. M.J.D. Powel. An efficient method for finding the minimum of a function of several variables without calculating derivatives. The Computer Journal, 7:155-162, 1964.

14. W T Vetterling Press W, S A Teukolsky and B P Flannery. Numerical Recipes (any edition). Cambridge University Press.

15. P.L. Rosin. Shape partitioning by convexity. IEEE Transactions on Systems, Man, and Cybernetics - Part A: Systems and Humans, 30(2):202-210, mar 2000.

16. Oliver Schmitt and Maria Hasse. Morphological multiscale decomposition of connected regions with emphasis on cell clusters. Comput. Vis. Image Underst., 113(2):188-201, February 2009.

17. Sopohie Schüpp, Abderrahim Elmoataz, Jalal M. Fadili, and Daniel Bloyet. Fast statistical level sets image segmentation for biomedical applications. In Third International Conference Scale-Space 2001, volume 2106, pages 308-388, Vancouver, Canada, 2001.

18. J. Serra. Image analysis and mathematical morphology. Lon: Academic Press, 1982.

19. P. Soille. Morphological image analysis: principles and applications. Secaucus, NJ, USA, 2003. 
20. H Talbot and B. C. Appleton. Elliptical distance transforms and object splitting. International Symposium on Mathematical Morphology, pages 229-240, April 2002. 